\title{
Experimentelle Untersuchungen zu kombinierten hydraulisch-mechanischen Gesteinslöseprozessen
}

\author{
Thomas Stoxreiter und Robert Galler \\ Lehrstuhl für Subsurface Engineering, Dept. Mineral Resources Engineering, Montanuniversität Leoben, Leoben, \\ Österreich
}

Eingegangen 15. Oktober 2019; angenommen 16. Oktober 2019; online publiziert 5. November 2019

\begin{abstract}
Zusammenfassung: Im Zuge des von der EU geförderten Projektes „ThermoDrill“ wurde eine alternative Bohrtechnologie für Anwendungen in der Tiefengeothermie, mit dem Ziel eines schnelleren Bohrfortschrittes, entwickelt. Das alternative Bohrverfahren verwendet eine Kombination aus Hochdruckfluidstrahlen mit mechanischem Rotationsbohren. Im Rahmen der Entwicklung dieses Bohrverfahrens wurden umfassende experimentelle Studien zur Untersuchung des kombinierten hydraulisch-mechanischen Löseprozesses durchgeführt. Die möglichst realitätsnahe Simulation der Umgebungsdruckbedingungen während der Versuche war dabei eines der zentralen Kriterien. Der positive Effekt einer durch den Hochdruckstrahl erzeugten Kerbe auf den mechanischen Lösevorgang wurde zunächst im kleineren Maßstab nachgewiesen. Großmaßstäbliche Bohrversuche im Labor bestätigten sowohl anhand des erhöhten Bohrfortschrittes als auch anhand der Auswertung der Mikrorissbilder in der Bohrlochsohle der Gesteinsproben die Ergebnisse aus den kleinmaßstäblichen Versuchen.
\end{abstract}

Schlüsselwörter: Hochdruckfluidstrahlen, Bohrtechnik, Felsmechanik

Experimental Investigations Concerning Combined Hydraulic-mechanical Rock Destruction Processes

Abstract: The purpose of the EU funded project "ThermoDrill" was the development of an alternative drilling technology, drilling faster than conventional systems, for deep geothermal applications. The alternative drilling system is based on the combination of high-pressure fluid jetting and rotary drilling. Comprehensive experimental studies concerning the combined hydraulic-mechanical rock destruc-

\footnotetext{
T. Stoxreiter $(\bowtie)$

Lehrstuhl für Subsurface Engineering, Dept. Mineral Resources Engineering,

Montanuniversität Leoben,

Erzherzog Johann Straße 3,

8700 Leoben, Österreich

thomas.stoxreiter@unileoben.ac.at
}

tion process were conducted. To capture the effects of increased ambient pressure conditions during the experiments was a major task. The positive impact of the jetted kerf on the mechanical rock destruction process was firstly verified in scaled laboratory experiments. Full-scale drilling experiments confirmed the results of the scaled laboratory experiments via the increased drilling performance as well as via the evaluation of the micro crack pattern in the hole bottom of the drilled rock specimens.

Keywords: High-pressure fluid jetting, Drilling, Rock Mechanics

\section{Einleitung}

Eine zuverlässige Versorgung Europas mit nachhaltiger Energie ist eine der aktuell größten Herausforderungen der Europäischen Union. Der Etablierung eines sicheren, wirtschaftlichen und nachhaltigen Energiesystems wird dabei hohe Priorität eingeräumt. Dabei wird die Nutzung von geothermischer Energie, speziell in Form von EGS (Enhanced Geothermal Systems), als zukünftiger Eckpfeiler der europäischen Strategie für erneuerbare Energieformen betrachtet. Allerdings liegen passende geothermische Reservoire zumeist in großer Tiefe, weshalb die Bohrkosten einen großen Teil der Gesamtkosten für EGS Projekte betragen. Eine deutliche Erhöhung der Bohrgeschwindigkeit (Rate of Penetration, ROP) ist eine Option, um die Bohrkosten maßgeblich zu senken. Aus diesem Grund wurde im Rahmen des von der EU geförderten Forschungsprojekts „ThermoDrill“ eine alternative Bohrtechnologie entwickelt, welche eine signifikante Steigerung des Bohrfortschrittes erlaubt. Das Projektkonsortium bestand aus neun Partnerorganisationen aus sechs europäischen Ländern, wobei von der Montanuniversität Leoben neben dem Lehrstuhl für Subsurface Engineering das Außeninstitut und der Lehrstuhl für Petroleum and Geothermal Energy Recovery aktiv am Projekt beteiligt waren. Das Projekt ist im Detail in Stoxreiter et al. [1] beschrieben. 


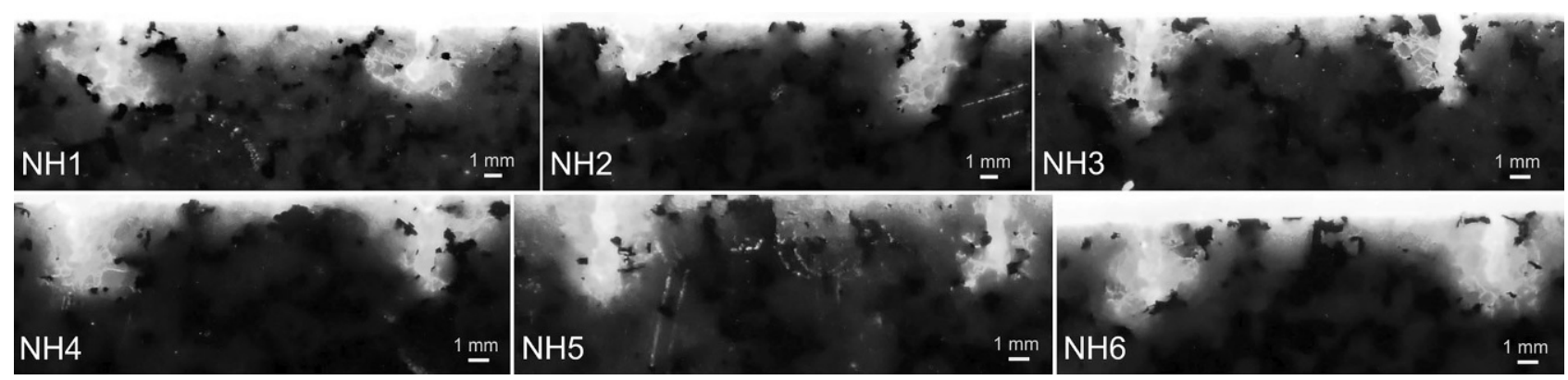

Abb. 1: Mit fluoreszierendem Harz gefüllte Risse und Kerbe entlang der Schneidspur eines Hochdruckwasserstrahls mit 4000 bar Staudruck, 0,25 mm Düsendurchmesser und rund $13 \mathrm{~kW}$ hydraulischer Leistung unter atmosphärischen Bedingungen [8]

Die grundlegende Idee des Projektes war die Entwicklung einer alternativen Bohrtechnologie durch die Kombination von konventionellem Rotationsbohren und Hochdruckfluidstrahlen. Diese Idee existiert bereits seit Jahrzehnten. Einer der ersten Ansätze wurde 1963 von Bobo in den USA patentiert [2]. In dem patentierten Verfahren wird ein Druckübersetzer im Bohrstrang installiert, welcher den Hochdruck im Bohrloch erzeugt. Der Hochdruck wird dann durch den Bohrmeißel zu den Hochdruckdüsen transportiert und schneidet schließlich als Strahl eine Kerbe im äußeren Bereich der Bohrlochsohle. Der innere Bereich der Bohrlochsohle sollte dann einfacher zu bohren sein, was wiederum einen deutlich höheren Bohrfortschritt erlaubt. Die meisten der nachfolgenden Konzepte, wie auch jene von Veenhuizen et al. [3] oder Shi et al. [4], basieren im Grunde auf dieser ersten Idee von Bobo, wobei die technische Umsetzung sich in wichtigen Aspekten wie etwa der Art der Hochdruckerzeugung, dem Bohrmeißeldesign und der Düsenkonfiguration unterscheidet.

Zur Untersuchung der zugrundeliegenden Mechanismen des Gesteinslöseprozesses in Verbindung mit der Rissinitiierung und Rissausbreitung aufgrund der kombinierten hydraulischen und mechanischen Einwirkung wurden umfassende experimentelle Studien unter simulierten Bohrlochbedingungen durchgeführt. Zusätzlich wurden großmaßstäbliche Bohrversuche und Feldtests im Rahmen des Projekts erfolgreich abgewickelt.

\section{Hydraulisches Gesteinslösen mit Hoch- druckfluidstrahlen}

Obwohl bisher sehr viel Forschung im Bereich des Hochdruckwasserstrahlschneidens von Gestein betrieben wurde, sind nur wenige genauere Untersuchungen zur Schneidleistung der Hochdruckstrahlen unter simulierten Bohrlochdruckbedingungen bekannt. Die meisten Versuche wurden unter atmosphärischen Bedingungen durchgeführt, welche sich allerdings komplett von den Verhältnissen an der Bohrlochsohle unterscheiden. Nur vereinzelt wurden Anwendungen von Hochdruckstrahlen unter beträchtlichem Gegendruck berücksichtigt. Als Gegendruck wird der Druck in der Fluidschicht zwischen dem Düsenaustritt und dem zu schneidenden Objekt definiert. Reichmann [5] hat repräsentative Schneidversuche an Granit mit Hochdruckfluidstrahlen durchgeführt, wobei der maximale Gegendruck ca. $207 \mathrm{bar}$, der maximale Düsendurchmesser $0,45 \mathrm{~mm}$ und der maximale Staudruck ca. 3100 bar betragen hat. Er konnte dabei beobachten, dass die Schneidwirkung des Strahls beim maximalen Gegendruck von ca. 207 bar völlig zum Erliegen kam. Kolle [6] machte ähnliche Beobachtungen bei seinen Experimenten mit Sedimentgesteinen. Da beide Quellen von einem vollkommenen Einstellen der Schneidleistung ab einem mittleren bis hohen Gegendruck berichten, musste die generelle Umsetzbarkeit dieser alternativen Bohrtechnik für die erwarteten Bedingungen bei tiefen Geothermie-Projekten hinterfragt werden, falls keine ausreichende Schneidleistung erzielt werden konnte. Um diese Unklarheiten zu beseitigen, wurden umfassende Schneidversuche unter verschiedenen Druck-Randbedingungen und mit unterschiedlichen Strahlparametern durchgeführt [7].

Da Bohrungen für EGS Projekte zumeist mehrere Kilometer in hartem, kristallinem Gebirge abgeteuft werden müssen, erfolgten die Versuche hauptsächlich mit Neuhauser Granit als repräsentativem Vertreter kristalliner Gesteine. Die gemessene Permeabilität des Neuhauser Granits beträgt zwischen $0,05 \mathrm{mD}$ und $0,00 \mathrm{mD}$. Nachdem die Abmessungen der geschnittenen Kerbe mittels eines Lasersensors ermittelt wurden, wurden die Gesteinsproben in einem Exsikkator unter Vakuum mit einem fluoreszierenden Harz getränkt. Nach dem Aushärten des Harzes wurden die Gesteinsproben in ca. 2-3 mm dicke Scheiben geschnitten und unter UV-Licht das Rissmuster untersucht. Mit Hilfe des Farbeindringverfahrens wurde stichprobenartig überprüft, ob noch unverfüllte Risse vorhanden sind, was nicht der Fall war [8]. Die Experimente haben gezeigt, dass die Schneidleistung des Hochdruckstrahls mit erhöhtem Gegendruck signifikant abnimmt. Die wesentlichen Strahlparameter sind dabei der Abstand der Düse vom Gestein und die hydraulische Leistung des Hochdruckfluidstrahls [7]. Wie bereits von Rehbinder [9] festgestellt wurde, haben sowohl die Korngröße als auch die Permeabilität des Gesteins einen Einfluss auf die Schneidleistung des Hochdruckstrahls. Die Form der Kerbe hängt von den geschnittenen Mineralen ab, Schichtsilikate etwa brechen zumeist transkristallin, während Quarzminerale sowohl trans- als auch interkristalline Risse aufweisen. Wie in Abb. 1 zu sehen ist, weisen die Gesteinsproben um die Kerben herum ein rund $2 \mathrm{~mm}$ weit verzweigtes Rissnetzwerk auf. Abb. 1 zeigt mehrere Querschnitte der Kerbe entlang der Schneidspur unter atmosphärischen Bedingungen. Das 


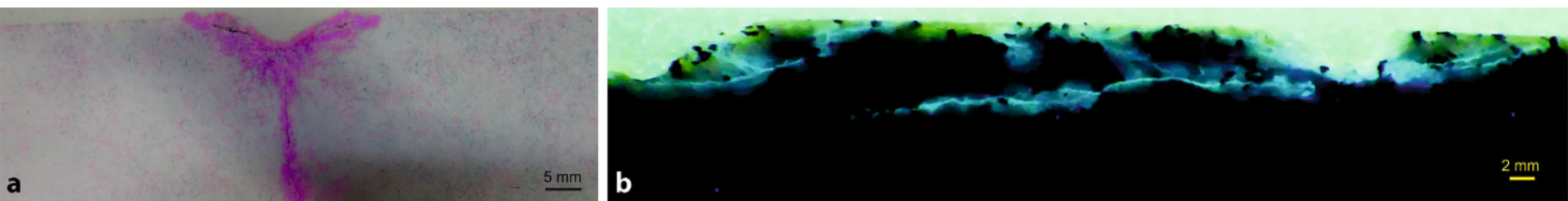

Abb. 2: a Mit Farbeindringverfahren sichtbar gemachter vertikaler Zugriss unterhalb eines Kraters aus einem Eindrückversuch ohne benachbarte freie Oberfläche. b Ausgeprägte subhorizontale Risse vom Krater des Eindrückversuchs (rechts) in Richtung der Kerbe (links) [8]

Ausmaß dieses Rissnetzwerks hängt stark von der hydraulischen Leistung des Strahls und vom Umgebungsdruck ab.

\section{Versuche mit Hartmetalleinsätzen für Rollenmeißel}

Um die Interaktion von mittels Hochdruckfluidstrahlen erzeugten Kerben mit dem mechanischen Lösevorgang von Hartmetalleinsätzen für Rollenmeißel zu untersuchen, wurden Eindrückversuche durchgeführt. Dabei wurde ein sogenannter "bullet-shaped“ Hartmetalleinsatz für Rollenmeißel in variierenden Abständen zu bereits geschnittenen Kerben unterschiedlich tief in das Gestein eingedrückt. Die resultierenden Kräfte auf den Hartmetalleinsatz wurden mit hoher Abtastrate gemessen sowie das gelöste Volumen je Eindrückvorgang fotogrammetrisch ermittelt. Die Versuche wurden bei einem hydrostatischen Umgebungsdruck von 300 bar durchgeführt, wobei die Gesteinsproben vorab bei identem Umgebungsdruck mittels Hochdruckfluidstrahl gekerbt wurden. Danach wurden die Risse wiederum mit fluoreszierendem Harz und dem Farbeindringverfahren gesättigt und die Proben in Scheiben geschnitten. In Abb. 2a ist exemplarisch ein Rissmuster in einer Gesteinsprobe ohne Kerbe oder Krater von vorhergehenden Eindrückversuchen, sichtbar gemacht mittels Farbeindringverfahren, dargestellt. In Abb. 2b ist ein Rissmuster im Einflussbereich einer Kerbe, visualisiert mittels fluoreszierendem Harz, abgebildet. Bei Eindrückversuchen an Gesteinsproben ohne Kerbe traten ab einer gewissen Eindrücktiefe des Hartmetalleinsatzes die charakteristischen vertikalen Zugrisse auf vereinzelt noch zusätzliche subhorizontale Risse. Im Gegensatz dazu traten bei Eindrückversuchen im Einflussbereich der Kerbe primär subhorizontale Risse in Richtung der Kerbe auf. Die Rissbildung kam oftmals kurz vor der Kerbe zum Erliegen, was in Übereinstimmung mit den Ergebnissen numerischer Simulationen ist [10]. Während allerdings die vertikalen Zugrisse keinen relevanten Beitrag zum Lösen des Gesteins leisten, ermöglichen die subhorizontalen Risse in Richtung der Kerbe das Lösen eines viel größeren Volumens, was wiederum die Effizienz des gesamten Bohrvorganges deutlich erhöht. Da bei einem Umgebungsdruck von 300 bar der Hochdruckfluidstrahl keine Rissbildung um die Kerbe verursacht hat, ist diese ganz allgemein als freie Oberfläche zu betrachten. Die Versuche zeigen außerdem, dass die spezifische Energie für das Lösen mittels Hochdruckfluidstrahl deutlich größer ist als für mechanische $\mathrm{Ge}$ steinslöseprozesse [8].

\section{Großmaßstäbliche Bohrversuche im Labor}

Basierend auf den ermittelten Versuchsdaten wurden die Spezifikationen für den hydraulischen Teil des Bohrsystems festgelegt. Um diese Spezifikationen zu validieren und zusätzliche Informationen über die Leistung des neuen Bohrsystems unter realen Bohrbedingungen zu gewinnen, wurden großmaßstäbliche (8 1/2 Zoll Bohrmeißel Durchmesser) Laborversuche durchgeführt. Die Versuche wurden mit Hilfe des Bohr-Prüfstandes der Mines ParisTech in Pau, Frankreich durchgeführt (Abb. 3). Der Prüfstand ermöglicht großmaßstäbliche Versuche unter den simulierten Bedingungen einer tiefen Bohrung, wobei im Gegensatz zu einer realen Bohrung alle Parameter genau gesteuert und gemessen werden können. Im Allgemeinen wird dabei eine Gesteinsprobe, welche vergleichbaren Spannungszuständen wie im Bohrloch ausgesetzt ist, gebohrt. Die Überlagerungsspannung, der Umschlingungsdruck und der Porenwasserdruck werden über einen Drucktopf erzeugt. Zwei seitlich verlaufende Zylinder erzeugen die Vorschubkraft auf den Bohrmeißel (Weight on Bit, WOB). Der Bohrmeißel wird durch einen Elektromotor direkt am Prüfstand in die gewünschte Rotation versetzt. Der Motor ist dabei über ein Getriebe mit dem Bohrstrang verbunden. Mit Hilfe einer Pumpe wird die Bohrspülung bei definiertem Druck über den BohrmeiBel zur Gesteinsprobe und dem Ringraum transportiert, ein Ventil regelt den Ausflussdruck und damit auch den simulierten Überdruck an der Bohrlochsohle.

Da der ursprüngliche Prüfstand nicht für Versuche mit hochdruckfluidstrahl-unterstütztem Bohren ausgelegt war, wurden einige technische Modifizierungen und Ergänzungen am Prüfstand vom Konsortium vorgenommen. Der Hochdruck wurde mittels einer mobilen Hochdruckpumpe vom Typ URACA Jet Power 300-100, mit einer maximalen Flussrate von ca. $55 \mathrm{l} / \mathrm{min}$ bei 2500 bar, erzeugt. In der endgültigen Version des "ThermoDrill" Systems wird der Druck dann im Bohrloch mit Hilfe der neu entwickelten Druckerzeugungseinheit generiert. Da jedoch die Verifizierung aller relevanten Parameter vor der Produktion dieser Druckerzeugungseinheit erfolgen musste, wurde eine externe Hochdruckpumpe für die Versuche verwendet. Während der Versuche wurde die hydraulische Leistung des Hochdruckstrahls entsprechend den Ergebnissen aus den Schneidversuchen variiert [7]. Zwei innovative und zum Patent angemeldete Bohrmeißel-Prototypen, geplant und hergestellt von Smith Bits, A Schlumberger Company, wurden genauso getestet wie ein herkömmlicher Rollenmeißel. Für eine ideale Vergleichbarkeit der Ergebnisse hatten alle drei Bohrköpfe eine idente Schneidstruktur. Der herkömmliche Rollenmeißel ist für den Einsatz in harten 
Abb. 3: Übersicht über den Prüfstand für die großmaß stäblichen Versuche [11]

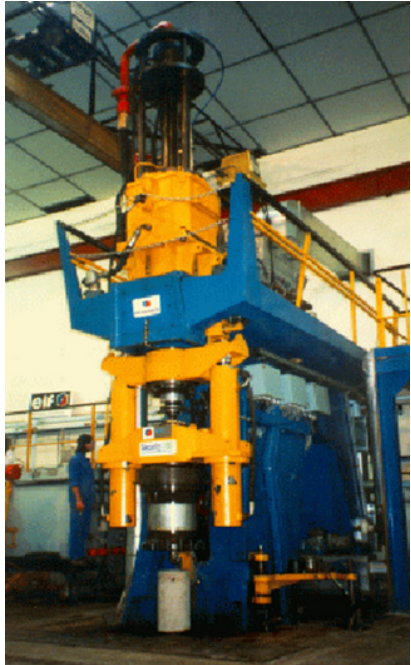

Formationen mit Hartmetalleinsätzen ausgestattet (IADC $627 Y$ ) und wird üblicherweise bei tiefen Bohrungen in Granit verwendet.

Ein wesentlicher Zweck der Versuche war, den Einfluss des Hochdruckstrahls auf die Bohrgeschwindigkeit (ROP) zu quantifizieren und mit jener des herkömmlichen Bohrmeißels zu vergleichen. Die Versuche zeigen eine signifikante Steigerung der ROP mit dem hochdruckstrahl-unterstützten Bohrsystem. Der Versuchsaufbau und die Ergebnisse sind im Detail in Stoxreiter et al. [11] beschrieben. Während bisher die Rissbildung und Rissausbreitung quasi getrennt für die hydraulischen und mechanischen Lösemethoden betrachtet wurden, tragen beide Mechanismen während der Bohrversuche simultan zum Gesteinslöseprozess bei. Um das entstandene Rissmuster untersuchen zu können, wurden Bohrkerne aus der Sohle der Gesteinsproben entnommen und entsprechend dem bereits vorgestellten Verfahren bearbeitet, um anschließend unter UVLicht die (Mikro-)Risse sichtbar zu machen. In Abb. 4 sind die Kerbe und der geschädigte Bereich, anhand der Mikrorisse, gut erkennbar. Der Druck an der Bohrlochsohle betrug während dieser Versuche rund $100 \mathrm{bar}$, weshalb die im oberen Bild sichtbaren Mikrorisse primär von der mechanischen Einwirkung der Hartmetalleinsätze stammen. Die Mikrorissstruktur weist Ähnlichkeit mit dem Rissbild aus den Eindrückversuchen auf. Wobei während des Bohrvorganges das Gestein im Bereich der Kerbe von mehreren Hartmetalleinsätzen nacheinander belastet wurde, was schlussendlich zum Ablösen eines Gesteinschips führt, wie im unteren Bild gut erkennbar ist. Aufgrund der signifikanten Vertiefung im normalerweise schwer lösbaren Bereich nahe dem Außenradius der Bohrlochsohle wurden die wirkenden Kräfte auf den inneren Bereich verlagert, was den deutlichen Anstieg der ROP im Vergleich zum herkömmlichen Bohrvorgang erklärt. Während die Struktur der Rissausbreitung in Abb. 4 exemplarisch für den Lösemechanismus von hochdruckstrahl-unterstütztem Bohren betrachtet werden kann, wurden auch Bereiche in der Bohrlochsohle mit weniger ausgeprägten Mikrorissen beobachtet. Dies liegt vermutlich daran, dass die Last auf den Meißel nach
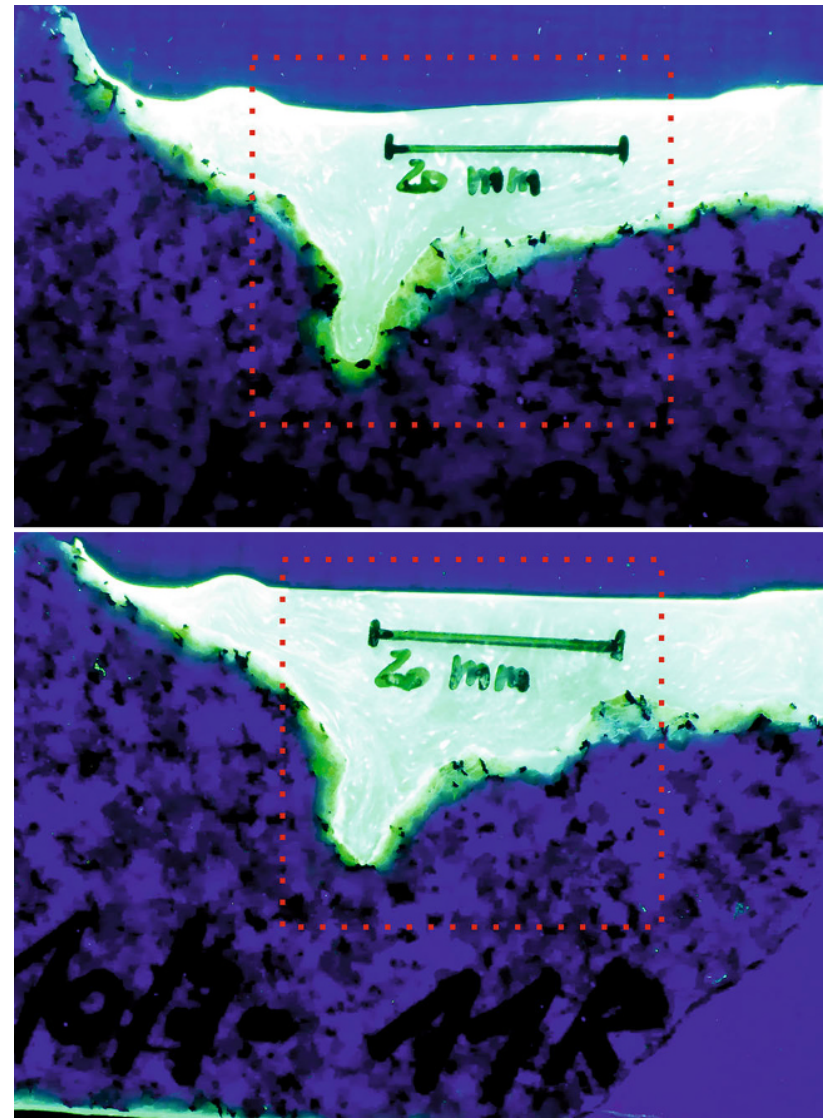

Abb. 4: Auswertung der induzierten Mikrorisse in der Bohrlochsohle einer Gesteinsprobe aus den großmaßstäblichen Bohrversuchen mit dem Rollenmeißel mit einem Hochdruckstrahl mit rund 2200 bar Staudruck und 1,3 mm Düsendurchmesser, gesättigt mit fluoreszierendem Harz [11]

Versuchsende relativ schnell reduziert wurde und daher in manchen Bereichen die mechanische Einwirkung der Hartmetalleinsätze zu gering war, um das Gestein in der Bohrlochsohle ausgeprägt zu schädigen.

\section{Zusammenfassung}

Im Rahmen des „ThermoDrill“ Projekts wurde eine alternative Bohrtechnologie für tiefe Geothermiebohrungen entwickelt. Erst der unermüdliche Einsatz aller Projektpartner hat das Erreichen dieses sehr ambitionierten Ziels ermöglicht. Die Technologie basiert auf einem hochdruckfluidstrahl-unterstützten Rotationsbohrsystem. Zur Untersuchung der kritischen Strahlparameter und um eine ausreichende Schneidleistung selbst unter hohen Umgebungsdruckbedingungen gewährleisten zu können, wurden umfassende experimentelle Studien durchgeführt. Weiters erfolgten Eindrückversuche unter erhöhten Umgebungsdruckbedingungen mit Hartmetalleinsätzen für Rollenmeißel. Der positive Effekt der mittels Hochdruckfluidstrahl erzeugten Kerbe als freie Oberfläche auf den mechanischen Lösevorgang wurde anhand mehrerer Indikatoren bestätigt. Großmaßstäbliche Bohrversuche haben die gesteigerte Bohrleistung des hochdruckstrahlunterstützten Bohrverfahrens in hartem kristallinem Ge- 
stein, im Vergleich zu herkömmlicher Rollenmeißeltechnik, bestätigt. Des Weiteren konnte durch Untersuchung der Mikrorisse in der Bohrlochsohle der Gesteinsproben aus den Versuchen auf den kombinierten hydraulisch-mechanischen Lösevorgang geschlossen werden.

Förderung. This project has received funding from the European Union's Horizon 2020 research and innovation programme under grant agreement no. 641202.

Funding. Open access funding provided by Montanuniversität Leoben.

Open Access Dieser Artikel wird unter der Creative Commons Namensnennung 4.0 International Lizenz (http://creativecommons.org/licenses/ by/4.0/deed.de) veröffentlicht, welche die Nutzung, Vervielfältigung, Bearbeitung, Verbreitung und Wiedergabe in jeglichem Medium und Format erlaubt, sofern Sie den/die ursprünglichen Autor(en) und die Quelle ordnungsgemäß nennen, einen Link zur Creative Commons Lizenz beifügen und angeben, ob Änderungen vorgenommen wurden.

\section{Literatur}

1. Stoxreiter, T.; Rehatschek, K.; Hofstätter, H.: ThermoDrill-Development of an alternative drilling technology for deep geothermal applications, World of Mining - Surface and Underground, 71 (2019), Nr. 5 S. 276-282

2. Bobo, R.: Method of drilling with high velocity jet cutter rock bit. Patent. United States Patent Office, 1963

3. Veenhuizen, S.D.; Stang, D.L.; Kelley, D.P.; Duda, J.R.; Aslakson, J.K.: Development and Testing of Downhole Pump for High-Pressure JetAssist Drilling, SPE Annual Technical Conference and Exhibition, San Antonio, 5-8 October 1997, SPE 38581, 1997
4. Shi, H.; Li, G.; Huang, Z.: Mechanism of hydraulic pulsed and cavitating jet improving ROP and application in China offshore drilling. WJTA-IMCA Conference and Expo, Houston, 9-11 September 2013, WJTA Water Jet Technology Association: Conference Proceedings, 2013

5. Reichman, J.M.: Research and development of a high-pressure waterjet coring device for geothermal exploration and drilling, Report for U.S. Department of Energy, 1977

6. Kolle, J.J.: Jet kerfing parameters for confined rock, Fourth U.S. Water Jet Conference, Berkeley, CA, 26-28 August 1987, WJTA: Proceedings, 1987, pp 134-144

7. Stoxreiter, T.; Martin, A.; Teza, D.; Galler, R.: Hard rock cutting with high-pressure jets in various ambient pressure regimes, International Journal of Rock Mechanics and Mining Sciences, Vol. 108 (2018), pp 179-188

8. Stoxreiter, T.; Wenighofer, R.; Portwood, G.; Pallesi, S.; Bertini, A.; Galler, R.; Grafinger, S.: Rock fracture initiation and propagation by mechanical and hydraulic impact, Open Geosciences, Akzeptiert, 2019

9. Rehbinder, G. A: Theory About Cutting Rock With a Water Jet, Felsmechanik, 12 (1980), S. 247-257

10. Huang, H.; Detournay, E.; Discrete element modeling of tool-rock interaction II: rock indentation, International Journal for Numerical and Analytical Methods in Geomechanics, Vol. 37 (2013), https://doi. org/10.1002/nag.2114

11. Stoxreiter, T.; Portwood, G.; Gerbaud, L.; Seibel, O.; Essl, S.; Plank, J.; Hofstätter, H.: Full-scale experimental investigation of the performance of a jet-assisted rotary drilling system in crystalline rock, International Journal of Rock Mechanics and Mining Sciences, Vol. 115 (2019), pp 87-98

Hinweis des Verlags. Der Verlag bleibt in Hinblick auf geografische Zuordnungen und Gebietsbezeichnungen in veröffentlichten Karten und Institutsadressen neutral. 